\title{
Enzyme-responsive nanocomposites for wound infection prophylaxis in burn management: in vitro evaluation of their compatibility with healing
} processes

\author{
This article was published in the following Dove Press journal: \\ International Journal of Nanomedicine \\ 24 June 2015 \\ Number of times this article has been viewed
}

\author{
Verena Grützner' \\ Ronald E Unger' \\ Grit Baier ${ }^{2}$ \\ Lars Choritz ${ }^{3}$ \\ Christian Freese ${ }^{1}$ \\ Thomas Böse' \\ Katharina Landfester ${ }^{2}$ \\ C James Kirkpatrick' \\ 'REPAIR-Lab, Institute of Pathology, \\ University Medical Center, Mainz, \\ 2Max Planck Institute for Polymer \\ Research, Mainz, Germany; \\ ${ }^{3}$ Department of Ophthalmology, \\ University Clinic, Magdeburg, \\ Germany
}

\begin{abstract}
Responsive, theranostic nanosystems, capable of both signaling and treating wound infections, is a sophisticated approach to reduce the most common and potentially traumatizing side effects of burn wound treatment: slowed wound healing due to prophylactic anti-infective drug exposure as well as frequent painful dressing changes. Antimicrobials as well as dye molecules have been incorporated into biodegradable nanosystems that release their content only in the presence of pathogens. Following nanocarrier degradation by bacterial enzymes, any infection will thus emit a visible signal and be effectively treated at its source. In this study, we investigated the effect of fluorescent-labeled hyaluronan nanocapsules containing polyhexanide biguanide and poly-L-lactic acid nanoparticles loaded with octenidine on primary human dermal microvascular endothelial cells, which play a major role in cutaneous wound healing. Microscopic and flow cytometric analysis indicated a time-dependent uptake of both the nanocapsules and the nanoparticles. However, enzyme immunoassays showed no significant influence on the expression of pro-inflammatory cell adhesion molecules and cytokines by the endothelial cells. Under angiogenic-stimulating conditions, the potential to form capillary-like structures in coculture with dermal fibroblasts was not inhibited. Furthermore, cytotoxicity studies (the MTS and crystal violet assay) after short- and long-term exposure to the materials demonstrated that both systems exhibited less toxicity than solutions of the antiseptic agents alone in comparable concentrations. The results indicate that responsive antimicrobial nanocomposites could be used as an advanced drug delivery system and a promising addition to current best practice wound infection prophylaxis with few side effects.
\end{abstract}

Keywords: enzyme-responsive nanosystems, burn wound infection, endothelial cells, wound healing

\section{Introduction}

Bacterial infections represent a ubiquitous problem in wound management. While localized infections cause tissue destruction and necrosis, often leading to cosmetic as well as functional impairment as late sequelae, wound infections can also develop into acutely life-threatening systemic forms such as sepsis. Despite the extensive efforts focused on both the prevention and treatment of infections, they are still considered as the most serious wound healing disorder and remain a major challenge in health care.

Among cutaneous lesions, thermic injury occupies a special position. Predisposing factors including the condition and extent of burn wounds make them particularly
Correspondence: Verena Grützner REPAIR-Lab, Institute of Pathology, University Medical Center, Langenbeckstraße I, 55I3I Mainz, Germany

Email vgruetzn@uni-mainz.de (c) (i) (\$) 2015 Grützner et al. This work is published by Dove Medical Press Limited, and licensed under Creative Commons Attribution - Non Commercial (unported, v3.0) License. The full terms of the License are available at http://creativecommons.org/licenses/by-nc/3.0/. Non-commercial uses of the work are permitted without any further permission from Dove Medical Press Limited, provided the work is properly attributed. Permissions beyond the scope of the License are administered by Dove Medical Press Limited. Information on how to request permission may be found at: http://www.dovepress.com/permissions.php 
susceptible for infections so that early anti-infective therapy is indicated. ${ }^{1,2}$ According to the gold standard, general disinfection measures, combined with a close monitoring of the wound following debridement, are implemented in order to reduce the risk of infection. However, mechanical manipulations caused by dressing changes are extremely painful for the patient and the use of antiseptic agents may lead to irritation and affect healing processes. Silver sulfadiazine, for example, is widely used for the disinfection of burns, but it is also known to have a negative impact on re-epithelialization. ${ }^{3}$ In addition, as a sulfonamide derivative it promotes the development of bacterial resistance. ${ }^{4,5}$ Other types of drug delivery, such as nitrogen oxide-releasing wound dressings, were shown to have a significant inhibitory effect on microbes, but at the same time eukaryotic cell viability also decreased. ${ }^{6}$ Recent studies also indicate that nanoscaled systems utilizing the antimicrobial activity of heavy metals such as silver and zinc seem to be a suitable alternative to conservative disinfection measures. ${ }^{7,8}$ Nevertheless, the therapeutic activity of these drugs is also not selective and inhibitory effects are not limited to pathogens but may affect cells and tissue, too. ${ }^{9-12}$

By a combination of biological mimicry and nanotechnology, a novel approach to reduce such side effects has been developed. Antimicrobials have been incorporated into biodegradable polymeric nanosystems that release their content only in the presence of pathogens. Following the degradation of the nanosystem by bacterial enzymes, any infection will be effectively treated at its source and, if the nanocarrier is also loaded with dye molecules, the system will emit a visible signal on activation. This innovative approach could reduce the need for prophylactic disinfection measures, including regular traumatic dressing changes.

Fluorescent-labeled hyaluronan nanocapsules containing polyhexamethylene biguanide (HYA-PHMB-NC) ${ }^{13}$ as well as poly-L-lactic acid nanoparticles loaded with octenidine (PLLA-OCT-NP) ${ }^{14}$ have been previously described that display the above-named characteristics. Their susceptibility to degradation by hyaluronidase and proteinase facilitates a response to the most common germs found in infected burn wounds, such as Staphylococcus aureus and Pseudomonas aeruginosa. ${ }^{15}$ However, to ensure an adequate biocompatibility of the nanocomposites, potential effects on cells and tissue have to be extensively evaluated as well. Endothelial cells are among the first cells to come into contact with nanocompounds in general, not only in wounds, but also upon inhalation or injection. In addition, microvascular endothelial cells play a key role in wound healing processes such as inflammation and angiogenesis and therefore represent an important experimental model for this purpose.

The goal of the present study was to investigate the reaction of primary human dermal microvascular endothelial cells (HDMECs) to HYA-PHMB-NC and PLLA-OCT-NP in their non-degraded form simulating non-infectious conditions to evaluate their applicability in wounds. In addition to cytotoxicity assessment, we focused on nanocarrier uptake, inflammatory response, and effects on the potential to form capillary-like structures in vitro.

\section{Materials and methods Nanocomposites}

Nanocomposites were synthesized and characterized based on methodology described previously. ${ }^{13,14}$ However, for cell culture application, both samples were prepared using slightly modified procedures. Briefly, HYA-PHMB-NC were synthesized using the inverse mini-emulsion technique. They were purified by repetitive centrifugation $(3,300 \times g, 20$ minutes, two times) in order to remove surfactant residues and the pellet was redispersed in cyclohexane. The nanocapsules (NCs) were then transferred to an aqueous phase (Lutensol AT50, BASF, Ludwigshafen, Germany, aqueous solution $0.1 \mathrm{wt} \%$ ) and afterwards the $\mathrm{NC}$ were cleaned by repeated centrifugation $(1,467 \times \mathrm{g}$, 20 minutes, three times) in order to remove residues of the surfactant Lutensol AT50 and were finally redispersed in $0.9 \% \mathrm{NaCl}$ before being stored under gentle stirring conditions. For the PLLA-OCT-NP, the surface-active agent hydroxyethyl starch $(10 \%$, degree of molar substitution $=0.5$, molecular weight $=200,000 \mathrm{~g} \mathrm{~mol}^{-1}$; Fresenius Kabi, Bad Homburg, Germany) was used as a stabilizer. The nanoparticles (NPs) were cleaned by repeated centrifugation/ redispersion in demineralized water in order to remove residual surfactant. After the last redispersion step, NP were resuspended in $0.9 \% \mathrm{NaCl}$ and were stored under gentle stirring conditions. Prior to the in vitro studies, the nanocomposites were centrifuged $(1,500 \times g, 20$ minutes $)$ and redispersed in cell culture medium.

Dynamic light scattering was performed using a PSS Nicomp Particle Sizer 380 (Nicomp Particle Sizing Systems, Port Richey, FL, USA). Zeta potentials were measured in $10^{-3} \mathrm{M}$ potassium chloride solution, $\mathrm{pH} 6.8$ with a Zeta Nanosizer (Malvern Instruments, Malvern, UK). Scanning electron microscope (SEM) images were made with a Leo 1530 Gemini (Zeiss, Oberkochen, Germany).

Polyhexanide (polyhexamethylene biguanide, 20\% in $\mathrm{H}_{2} \mathrm{O}$ ) was purchased from Fagron, Barsbüttel, Germany. 
Octenidine was purchased from Bode Chemie, Hamburg, Germany. For the toxicity studies, a stock solution of $10 \%$ was prepared in 70\% ethanol (AppliChem, Darmstadt, Germany).

\section{Cell isolation and growth conditions}

HDMEC were isolated from healthy juvenile foreskin as previously described. ${ }^{16}$ Briefly, the cells were obtained from the tissue by enzymatic digestion and grown to confluence, after which a biomagnetic separation of HDMEC was performed. Human dermal fibroblasts (HDFs) were obtained as the negative fraction following endothelial cell isolation. HDMEC were then cultured in Endothelial Cell Basal Medium MV (PromoCell, Heidelberg, Germany) supplemented with 15\% fetal bovine serum (Invitrogen, Life Technologies, Carlsbad, CA, USA), 100 U/100 mg/mL Penicillin/Streptomycin (Invitrogen), $10 \mathrm{mg} / \mathrm{mL}$ sodium heparin (Sigma-Aldrich, St Louis, MO, USA), and $2.5 \mathrm{ng} / \mathrm{mL}$ basic fibroblast growth factor (Sigma-Aldrich). HDF were cultured in Dulbecco's Modified Eagle's Medium 4,500 mg glucose/mL (SigmaAldrich) containing 10\% fetal bovine serum (Invitrogen), and $2 \mathrm{mM}$ Glutamax I (Gibco, Life Technologies, Carlsbad, CA, USA) and $100 \mathrm{U} / 100 \mathrm{mg} / \mathrm{mL}$ penicillin/streptomycin (Invitrogen).

Cells were maintained under standard conditions $\left(37^{\circ} \mathrm{C}\right.$, $5 \% \mathrm{CO}_{2}$, saturated moisture in atmosphere) and used between passages 3 and 5 .

\section{Assessment of cytotoxicity}

Cytotoxicity of HYA-PHMB-NC and PLLA-OCT-NP as well as polyhexanide and octenidine solution was determined by the MTS and crystal violet assay after short- (24 hours) and long-term (7 days) exposure. Prior to cell seeding, growth surfaces were coated with $0.2 \%$ gelatin (Sigma-Aldrich) for 30 minutes at $37^{\circ} \mathrm{C}$. Confluent cultures of HDMEC were trypsinized and seeded into 96-well cell culture plates (TPP Techno Plastic Products, Trasadingen, Switzerland) at a density of $1.2 \times 10^{4}$ cells per well. After 18-24 hours, serial dilutions of the nanocarrier suspensions in fresh culture medium ranging from 0.3 to $1,000 \mu \mathrm{g}$ nanoparticle $/ \mathrm{mL}$ (resulting in antimicrobial concentrations of 0.00933-31.1 $\mu \mathrm{g}$ polyhexanide/mL and $0.0498-166 \mu$ g octenidine $/ \mathrm{mL}$, respectively) were prepared and added to the cells after removing the old medium. The antiseptic agents alone were adjusted to the respective concentrations and processed analogously.

After 24 hours and 7 days, the cytotoxicity assays were performed. Cell viability was quantified by the CellTiter $96 \mathrm{AQ}_{\text {ueous }}$ non-radioactive assay (Promega, Mannheim,
Germany). The medium was removed, the cells were washed with $0.2 \%$ bovine serum albumin (Sigma-Aldrich) in HEPES buffer, and then incubated with MTS solution 1:6 diluted in medium at $37^{\circ} \mathrm{C}$. After 60 minutes, the supernatants were collected in 96-well plates and the absorbance was measured by spectroscopy at $\lambda=492 \mathrm{~nm}$ (GENios plus multiplate reader, Tecan, Männedorf, Switzerland). The amount of cell DNA was determined by the crystal violet assay. The residual cell layers were washed three times with phosphate-buffered saline (PBS) and then fixed with methanol/ethanol (both AppliChem) (2:1) for 15 minutes, before a crystal violet staining was performed as previously described. ${ }^{17}$ Briefly, the cells were stained with $0.1 \%$ crystal violet (Merck, Darmstadt, Germany), washed with tap water, and dried at room temperature overnight before 33\% acetic acid (AppliChem) was added to remove the DNA-bound dye from the cells into the supernatant. The absorbance was measured by spectroscopy at $\lambda=600 \mathrm{~nm}$. The absorption of untreated cells was set as $100 \%$ viability or cell DNA.

\section{Uptake: microscopic and flow cytometric cell analysis}

To investigate cell-nanocarrier interactions of cells after exposure to the samples for various time periods, HDMEC were stained for CD31 and examined microscopically and also analyzed by flow cytometry. For microscopic analysis, the cells were trypsinized and seeded onto $13 \mathrm{~mm}$ gelatincoated Thermanox ${ }^{\mathrm{TM}}$ coverslips (Thermo Fisher Scientific, Waltham, MA, USA) placed into 24-well plates (TPP) at a density of $6 \times 10^{5}$ cells per well. After $18-24$ hours, the medium was changed by adding $0.1 \mathrm{mg} / \mathrm{mL}$ of HYA-PHMB-NC and PLLA-OCT-NP. Cells were exposed to NPs for 2, 8, 24, and 72 hours after which the cell layers were washed twice with PBS and subsequently fixed with $3.7 \%$ paraformaldehyde (Merck) for 15 minutes. After this, the cells were washed three times with PBS, followed by addition of $0.2 \%$ Triton X (Sigma-Aldrich) in PBS and incubation for 10 minutes to permeabilize the cells. Primary antibody mouse antihuman CD31 (Dako, Hamburg, Germany) was used in a 1:50 dilution in PBS containing $1 \%$ of bovine serum albumin (Sigma-Aldrich) and was allowed to incubate for 60 minutes, before the cells were washed three times with $0.05 \%$ Tween 20 (SERVA, Heidelberg, Germany) in PBS. Subsequently, secondary antibody (Alexa Fluor 488 and 594, Life Technologies, Carlsbad, CA, USA, 1:1,000 in 1\% bovine serum albumin in PBS) was added and incubated for 60 minutes. After washing three times with $0.05 \%$ Tween 20 in PBS, nuclear counterstaining was performed by adding DAPI (Sigma-Aldrich) $(1: 10,000$ 
dilution in PBS) for 5 minutes. After thorough washing with PBS, the coverslips were finally transferred to slides, mounted with Fluoromount-G (Southern Biotech, Birmingham, AL, USA), and examined using a Keyence BZ-9000 (Keyence, Osaka, Japan). Processing of images and quantification of fluorescence and nuclei were performed using the open source software ImageJ $1.47 \mathrm{n}$ (National Institutes of Health, Bethesda, MD, USA).

For flow cytometry, the cells were detached and seeded onto gelatin-coated six-well plates (TPP) at a density of $3 \times 10^{6}$ cells per well. After they had grown to confluence, the culture medium was changed by adding $0.1 \mathrm{mg} / \mathrm{mL}$ of the NPs. Analogous to the microscopy experiment, exposure was for 2, 8, 24, and 72 hours, before the cells were washed twice with PBS and trypsinized. The cells were resuspended in $500 \mu \mathrm{L} \mathrm{PBS}$, kept at $4^{\circ} \mathrm{C}$, and immediately analyzed using a FACSCalibur flow cytometer (Becton Dickinson, Franklin Lakes, NJ, USA). For each suspension, $2 \times 50,000$ events were assayed and data quantification was performed with the mean fluorescence intensities provided by region statistics.

\section{Inflammatory response: enzyme immunoassay and enzyme-linked immunosorbent assay}

To investigate the effect of HYA-PHMB-NC and PLLAOCT-NP on inflammatory response, the expression of various cell adhesion molecules (CAMs) and cytokines by microvascular endothelial cells was analyzed.

For the CAM enzyme immunoassays (EIAs), confluent cultures of HDMEC were detached and seeded onto gelatincoated 96-well plates at a density of $1.2 \times 10^{4}$ cells per well. After the cells had grown to confluence, the medium was removed and $0.1 \mathrm{mg} / \mathrm{mL}$ nanosamples were added in fresh medium. As a positive control, the cells were treated with $1 \mu \mathrm{g} / \mathrm{mL}$ lipopolysaccharide derived from Escherichia coli to induce pro-inflammatory processes. The E-selectin EIA was performed after 4 hours exposure as described previously. ${ }^{18}$ The stimulation period for the analysis of ICAM-1 and VCAM-1 was 24 hours. Briefly, after blocking endogenous peroxidases and unspecific binding sites, the primary antibodies were added, followed by the biotinylated secondary antibody (both 45 minutes) and streptavidin-biotinylated horseradish peroxidase complex (60 minutes). Subsequently, the substrate solution was added for 20 minutes, and the absorbance was measured by spectroscopy at $\lambda=492 \mathrm{~nm}$ (GENios plus multiplate reader, Tecan).

For the detection of IL-6, IL-8, and MCP-1, enzymelinked immunosorbent assays (ELISA, DuoSet ${ }^{\circledR}$, R\&D
Systems, Minneapolis, MN, USA) were used. HDMEC were trypsinized and seeded onto gelatin-coated six-well plates at a density of $3 \times 10^{5}$ cells per well. After the cells had grown to confluence, they were treated with $0.1 \mathrm{mg} / \mathrm{mL}$ nanosamples or $1 \mu \mathrm{g} / \mathrm{mL}$ E. coli lipopolysaccharide as a positive control for 24 hours. The assay was then performed according to the manufacturer's instruction using the culture supernatants in a 1:10 dilution.

For the evaluation of the assays, the data were normalized to unstimulated cells.

\section{Angiogenesis: co-culture conditions and immunofluorescence}

Under specific conditions such as in co-culture with stromal cells, endothelial cells spontaneously form capillary-like structures in vitro. ${ }^{19}$ To investigate the effects of the nanocarriers on angiogenesis (or capillary-like structure formation), HDMEC were co-cultured with HDF and exposed to the samples, after which the endothelial cells were stained for CD31 and the tubular networks evaluated. First, HDF were detached and seeded into culture dishes $\left(9.6 \mathrm{~cm}^{2}\right.$ growth area, Greiner Bio-one, Frickenhausen, Germany) at a density of $3 \times 10^{6}$ cells per dish in Endothelial Cell Basal Medium MV, supplemented as described previously. Following fibroblast attachment, HDMEC were added to the HDF at a similar density. After 18-24 hours incubation, the medium was removed and replaced with medium containing the nanosamples $(0.01 \mathrm{mg} / \mathrm{mL})$ in Endothelial Cell Basal Medium $\mathrm{MV}$, containing $2 \%$ fetal calf serum and the supplements described above. Cells were exposed to NP for 48 hours, then the culture supernatants were replaced with fresh medium and the cells cultured for another 7 days. The co-cultures were then washed twice with PBS and fixed with $3.7 \%$ paraformaldehyde for 15 minutes, before they were washed three more times with PBS. To permeabilize the cells, $0.2 \%$ Triton $\mathrm{X}$ in PBS was added for 10 minutes. Primary antibody mouse antihuman CD31 (1:50 in PBS containing 1\% of bovine serum albumin in PBS) was allowed to bind for 60 minutes, before the cells were washed with $0.05 \%$ Tween 20 in PBS three times. Secondary antibody (Alexa Fluor 488, 1:1,000 in $1 \%$ bovine serum albumin in PBS) was then added and incubated for 60 minutes. After three final washings with PBS, the co-cultures were analyzed and images were collected using a Keyence BZ-9000 (Keyence).

\section{Statistics}

All experiments were repeated using cells of at least three different, healthy donors. Statistical analysis was performed 
via one-way analysis of variance (Bonferoni's multiple comparison test) using GraphPad Prism ${ }^{\circledR} 5.04$ (GraphPad Software Inc., La Jolla, CA, USA).

\section{Results}

\section{Nanocomposites}

Both nanosystems were investigated in terms of particle size, size distribution, and morphology (Figure 1).

A homogeneous distribution of capsules/particles with sizes within the nanometer scale was obtained using the mini-emulsion process. Positive zeta potentials were obtained for the PLLA-hydroxyethyl starch NP, since octenidine is a cationic surface-active agent. The zeta potential of the redispersed and purified hyaluronic/polyhexanide-based NC was negative $(-15 \mathrm{mV})$ on account of the presence of carboxylic groups on the surface of the hyaluronic acid-based NC.
From SEM images, the collapse of the hyaluronic acid-based nanocapsule wall was a result of drying effects.

\section{Cytotoxicity of HYA-PHMB-NC and PLLA-OCT-NP}

To investigate the cytotoxicity of HYA-PHMB-NC and PLLAOCT-NP for endothelial cells, MTS cell viability and crystal violet DNA staining assays were performed after 24 hours and 7 days exposure periods. The effects of polyhexanide and octenidine, the antiseptic agents representing the active substances in the nanosystems, on the cells were also analyzed.

A 24-hour treatment with HYA-PHMB-NC resulted in no observable negative effect on cell viability, whereas exposure to a polyhexanide solution alone at the same concentration as present in the nanocomposite and arithmetically released upon degradation leads to a distinct decrease in cell viability
A

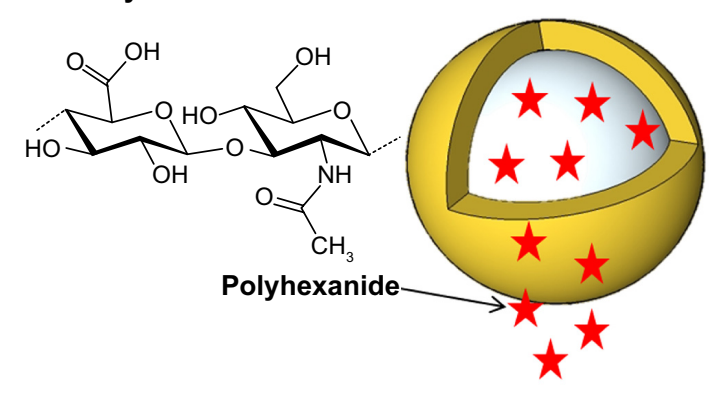

B

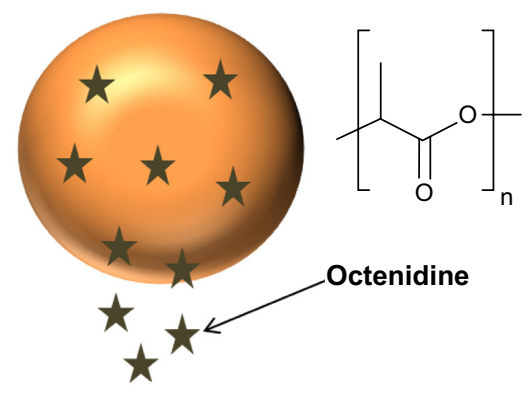

\begin{tabular}{lll}
\hline & HYA-PHMB-NC & PLLA-OCT-NP \\
\hline $\begin{array}{l}\text { Drug loading, } \\
\mu \mathrm{g} / \mathrm{mg} \text { nanosample }\end{array}$ & 31.1, polyhexanide & 166, octenidine \\
\hline $\begin{array}{l}\text { Size, } \mathrm{nm} / \mathrm{size} \\
\text { distribution, } \%\end{array}$ & $320 / 29$ & $230 / 24$ \\
\hline Zeta potential, $\mathrm{mV}$ & $-15 \pm 1$ & $+12 \pm 1$ \\
\hline
\end{tabular}
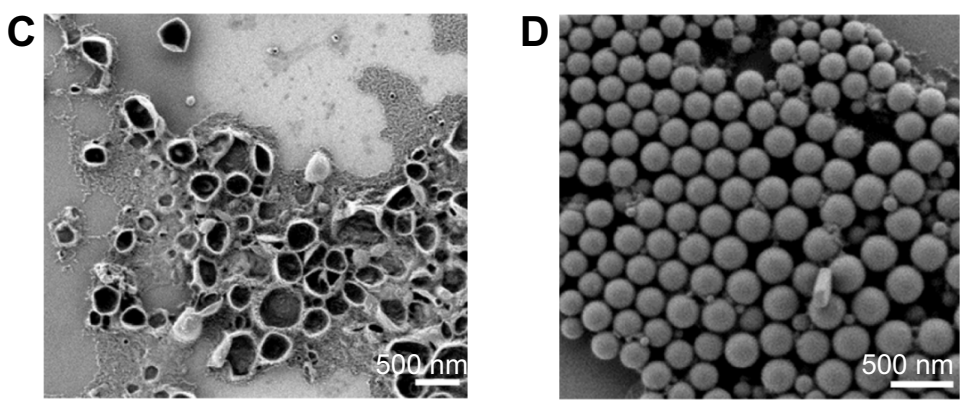

Figure I Physicochemical characteristics of HYA-PHMB-NC and PLLA-OCT-NP.

Notes: Schematic view of HYA-PHMB-NC (A) and PLLA-OCT-NP (B), drug loading, particle size, size distribution, zeta-potentials and SEM images of HYA-PHMB-NP (C) and PLLA-OCT-NP (D).

Abbreviations: SEM, scanning electron microscope; HYA-PHMB-NC, hyaluronan nanocapsules containing polyhexamethylene biguanide; PLLA-OCT-NP, poly-L lactic acid nanoparticles loaded with octenidine. 
(Figure 2A). In the crystal violet assay, the highest concentration tested ( $1 \mathrm{mg} \mathrm{NC} / \mathrm{mL}$ ) that is related to $31.1 \mu \mathrm{g} P H M B / m L$, HYA-PHMB-NC lead to a half-maximal decrease in the signal (Figure 2C). Still, like in the MTS cell viability assay, toxic effects caused by the polyhexanide solution at comparable concentrations started to appear one order of magnitude below (Figure 2C). After long-term exposure (7 days) (Figure 2B and D), effects on cells were observed in the presence of both the $\mathrm{NC}$ and the polyhexanide solution. However, the half-maximal inhibitory concentration $\left(\mathrm{IC}_{50}\right)$ of HYA-PHMB-NC was three (crystal violet) to seven (MTS) times higher than the $\mathrm{IC}_{50}$ of cells exposed to polyhexanide alone (Figure 2E). Over 24 hours, HYA-PHMB-NC concentrations $\leq 0.1 \mathrm{mg} / \mathrm{mL}(3.11 \mu \mathrm{g} / \mathrm{mL}$ PHMB) remained without any inhibitory effect on cells, over 7-day exposure, concentrations $\leq 0.01 \mathrm{mg} / \mathrm{mL}(0.311 \mu \mathrm{g} / \mathrm{mL}$ PHMB) were assessed as harmless. PLLA-OCT-NP exhibited only mild toxic effects after an exposure period of 24 hours, whereas octenidine solution alone at comparable concentrations affected the cells even at low concentrations (Figure 3A and C). Upon 7-day treatment, both the NP and the octenidine
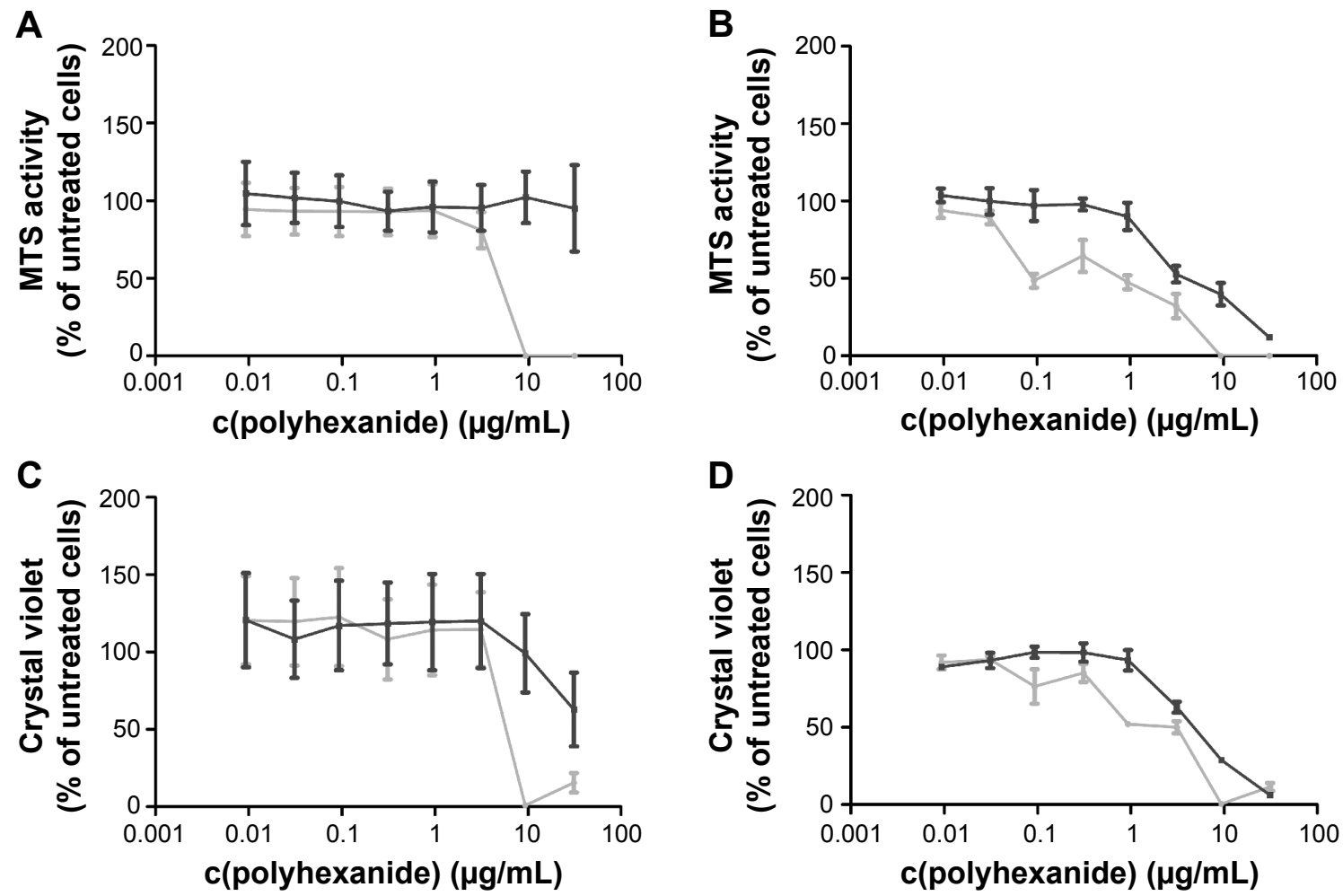

E

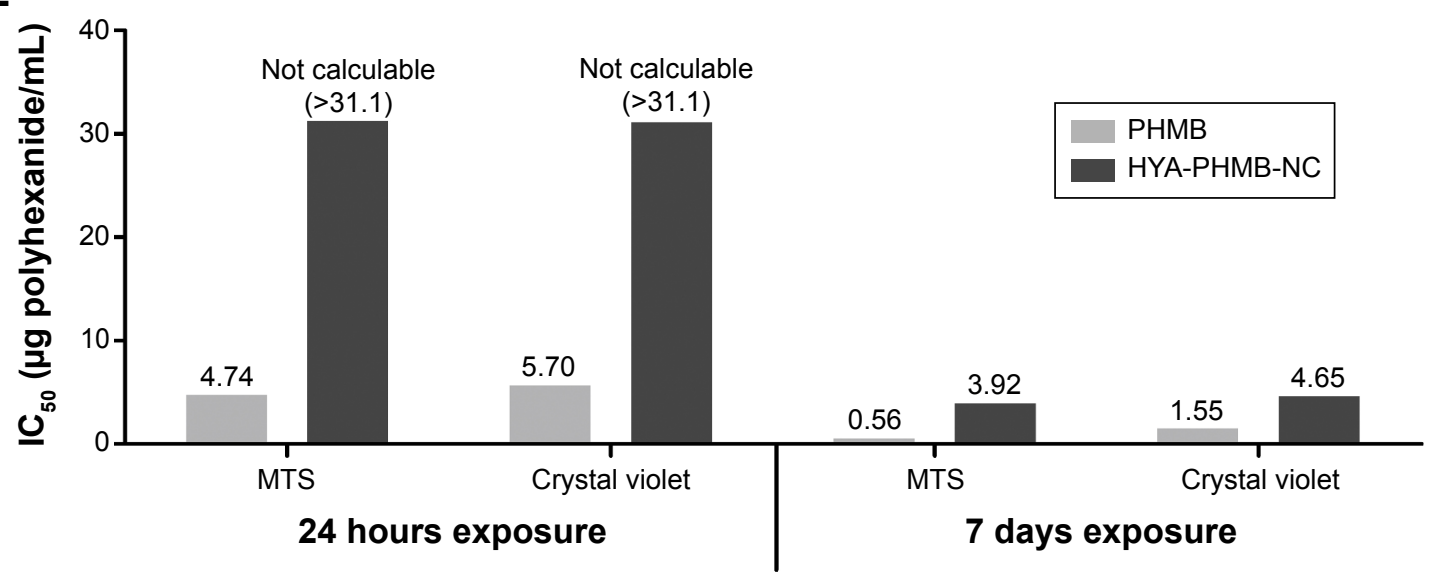

Figure 2 Toxicological assessment of HYA-PHMB-NC and polyhexanide.

Notes: MTS cell viability assay (A, B), crystal violet cell proliferation assay (C, D), and the IC $_{50}$ overview (E) after 24 hours (A, C) and 7 days (B, D) of exposure of HDMEC to the HYA-PHMB-NC show that the NC (lines and bars in dark gray) exhibit less toxic effects on the cells than polyhexanide solution (lines and bars in light gray) alone. $\mathrm{n}=3$.

Abbreviations: PHMB, polyhexamethylene biguanide; HYA-PHMB-NC, hyaluronan nanocapsules containing polyhexamethylene biguanide; HDMEC, human dermal microvascular endothelial cell; NC, nanocapsule; $I_{50}$, half-maximal inhibitory concentration; c(polyhexanide); concentration of polyhexanide. 
A
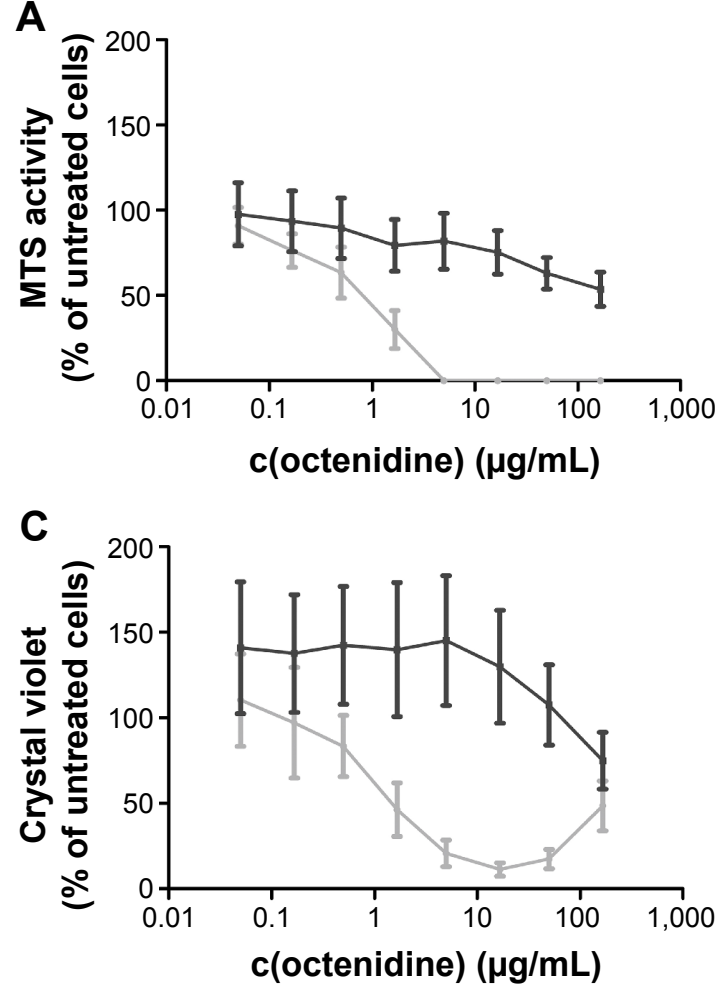

E
B

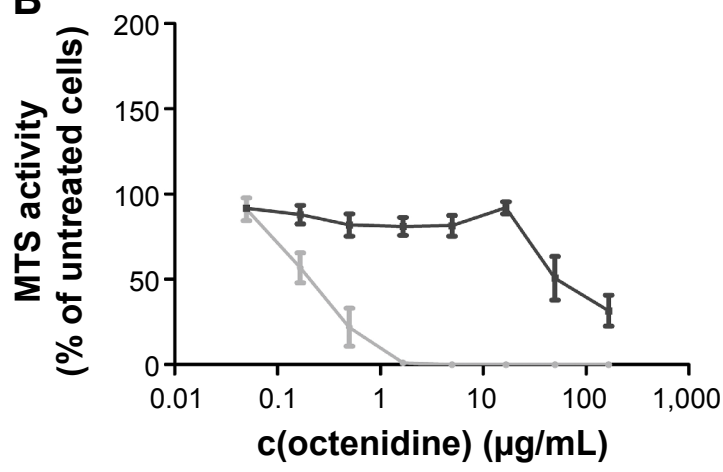

D

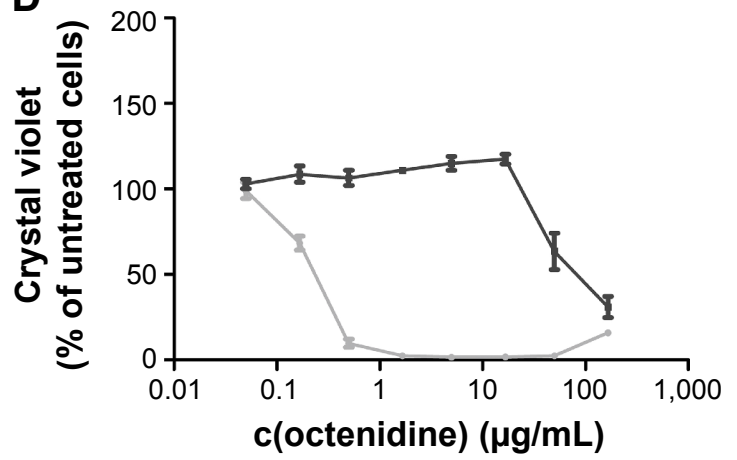

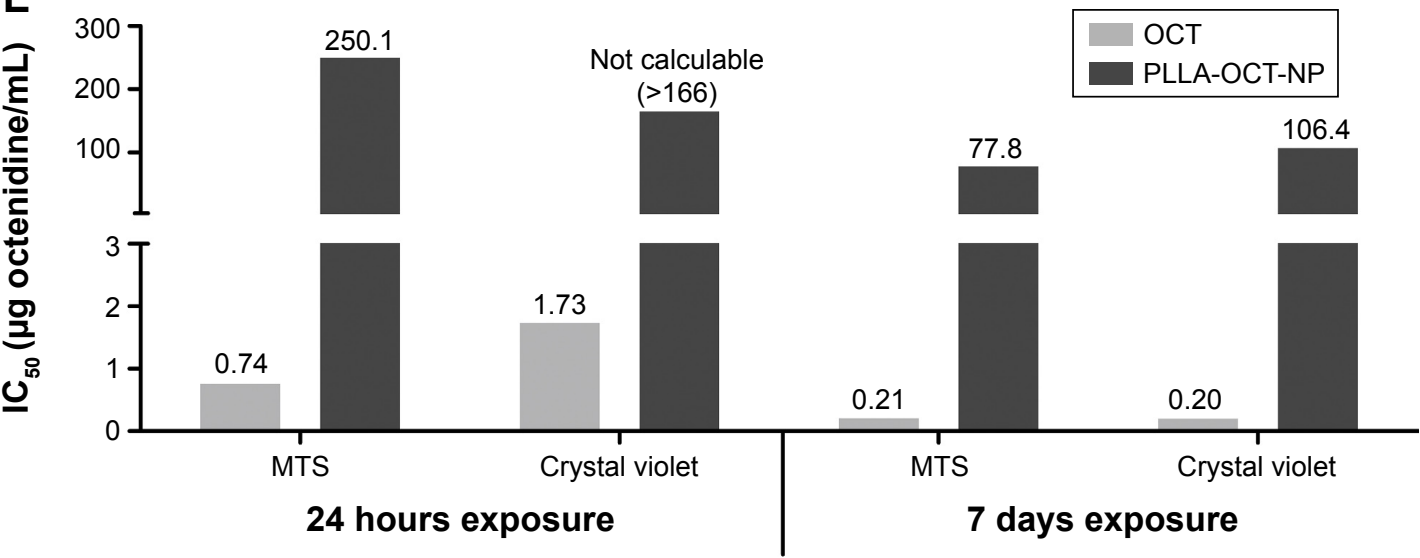

Figure 3 Toxicological assessment of PLLA-OCT-NP and octenidine.

Notes: The dose-response curves of the MTS cell viability assay $(\mathbf{A}, \mathbf{B})$ and crystal violet cell proliferation assay $(\mathbf{C}, \mathbf{D})$ after 24 hours $(\mathbf{A}, \mathbf{C})$ and 7 days (B, D) exposure of HDMEC to the PLLA-OCT-NP demonstrate that the NP (lines and bars in dark gray) were much less toxic than octenidine solution (lines and bars in light gray) alone. This is confirmed by the $\mathrm{IC}_{50}$ values $(\mathbf{E})$.

Abbreviations: OCT, octenidine; PLLA-OCT-NP, poly-L-lactic acid nanoparticles loaded with octenidine; HDMEC, human dermal microvascular endothelial cell; NP, nanoparticle; c(octenidine); concentration of octenidine.

solution lead to a decrease in cell viability (Figure $3 \mathrm{~B})$ and the amount of crystal violet and DNA, respectively (Figure 3D). However, the $\mathrm{EC}_{50}$ of PLLA-OCT-NP was 371 (MTS) to 532 (crystal violet) times higher than $\mathrm{IC}_{50}$ of octenidine (Figure $3 \mathrm{E}$ ), and nanoparticle concentrations $\leq 0.1 \mathrm{mg} / \mathrm{mL}(16.6 \mu \mathrm{g} / \mathrm{mL}$ OCT) exhibited no distinct effect on the cells.

\section{Internalization of HYA-PHMB-NC and PLLA-OCT-NP into endothelial cells}

To investigate cellular reactions to the nanosystems, endothelial cells were exposed to non-toxic concentrations
(0.1 mg/mL) of HYA-PHMB-NC and PLLA-OCT-NP for various periods (2, 8, 24, and 72 hours). Analysis included both immunofluorescence and flow cytometry. For quantification of the microscopic data, fluorescence intensity was normalized to the number of cells.

Microscopic images showed that the amount of HYAPHMB-NC (red) and associated with the cells increased over time (Figure 4A-D). For PLLA-OCT-NP (green), the amount of particles co-localized to the cells increased in a similar manner (Figure 5A-D). After 72 hours of exposure, the nanocarriers were primarily arranged in the perinuclear 

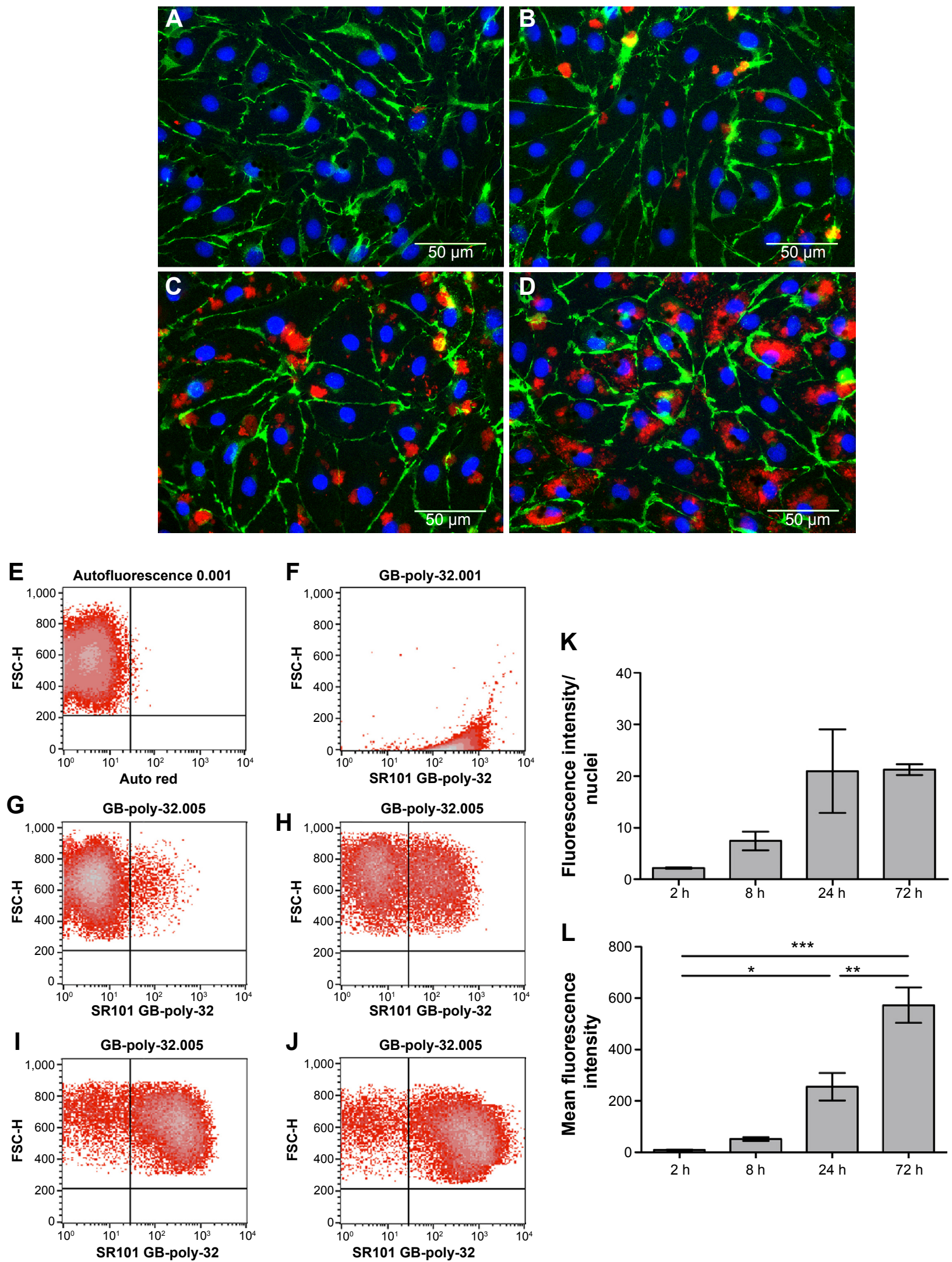

Figure 4 Endothelial cellular uptake of HYA-PHMB-NC.

Notes: (A-D; K) Microscopic analysis. HDMEC were stained for CD3I (green) and Hoechst (blue) after exposure to $0.1 \mathrm{mg} / \mathrm{mL}$ HYA-PHMB-NC (red) for 2 hours (A), 8 hours (B), 24 hours (C), and 72 hours (D). For data quantification, fluorescence intensity was normalized to nuclear fluorescence (K). (E-J; L) Flow cytometric analysis. Untreated HDMEC (E) and HYA-PHMB-NC alone (F) were used as controls. HDMEC were analyzed after exposure to 0.1 mg/mL HYA-PHMB-NC for 2 hours (G), 8 hours $(\mathbf{H}), 24$ hours (I), and 72 hours (J). Mean fluorescence intensity was used for quantification (L). $n=3, * P \leq 0.05, * * P \leq 0.01, * * * P \leq 0.00$ I.

Abbreviations: HYA-PHMB-NC, hyaluronan nanocapsules containing polyhexamethylene biguanide; HDMEC, human dermal microvascular endothelial cells; h, hour. 

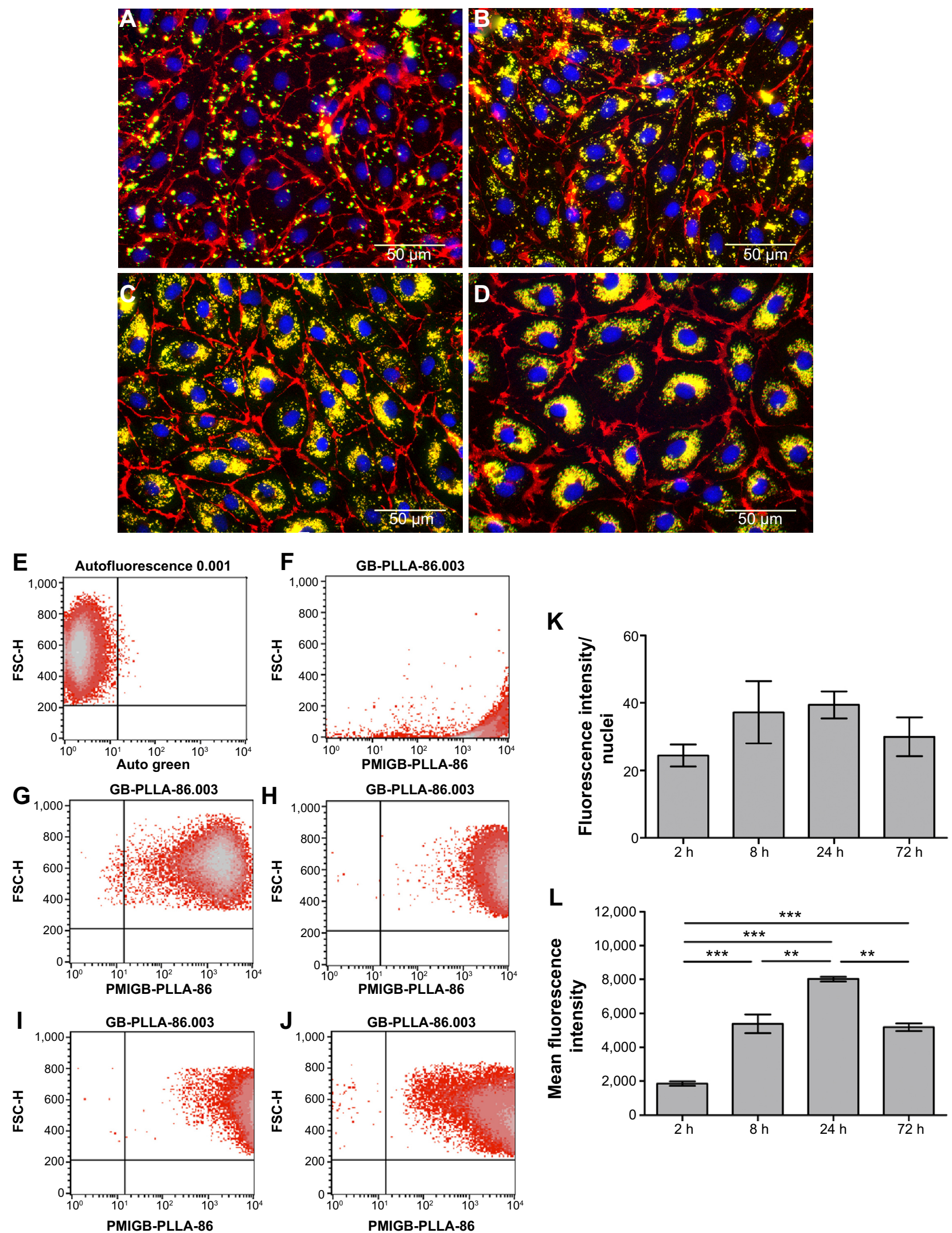

Figure 5 Endothelial cellular uptake of PLLA-OCT-NP.

Notes: (A-D; K) Microscopic analysis. HDMEC were stained for CD3I (red) and Hoechst (blue) after exposure to $0.1 \mathrm{mg} / \mathrm{mL}$ PLLA-OCT-NP (green) for 2 hours (A), 8 hours (B), 24 hours (C), and 72 hours (D). For data quantification, fluorescence intensity was normalized to nuclear fluorescence (K). (E-J; L) Flow cytometric analysis. Untreated HDMEC (E) and PLLA-OCT-NP alone (F) were used as controls. HDMEC were analyzed after exposure to 0.1 mg/mL PLLA-OCT-NP for 2 hours (G), 8 hours $(\mathbf{H}), 24$ hours (I), and 72 hours (J). Mean fluorescence intensity was used for quantification (L). $n=3, * * P \leq 0.01$, **** $\leq 0.00$ I.

Abbreviations: PLLA-OCT-NP, poly-L-lactic acid nanoparticles loaded with octenidine; HDMEC, human dermal microvascular endothelial cells; $h$, hour. 
region, indicating that they had not only adhered to the outer cell membrane, but also were internalized into the cytoplasm (Figures 4D and 5D). Data quantification confirmed the time dependence of the uptake processes (Figures $4 \mathrm{~K}$ and $5 \mathrm{~K}$ ). However, for the PLLA-OCT-NP-treated cells, the fluorescence intensity detected after 72 hours exposure was slightly lower than after 24 hours.

For flow cytometric analysis, the autofluorescence of untreated cells was used as the negative control and this was used to adjust the voltage of the photomultiplier tubes and to set the quadrant gates (Figures $4 \mathrm{E}$ and 5E). Dot plots of the nanocarriers alone showed no overlap with the cell population (Figures $4 \mathrm{~F}$ and $5 \mathrm{~F}$ ). Comparable to the microscopic data, flow cytometry of the cells after exposure to the nanosamples demonstrated a time-dependent increase in fluorescence due to an uptake of the fluorescent nanosystems (Figures 4G-J and 5G-J). Mean fluorescence intensity confirmed a differential and significant uptake over time as can be seen in the microscopic data quantification (Figures 4L and 5L).

\section{Inflammatory response on exposure to HYA-PHMB-NC and PLLA-OCT-NP}

To investigate the effects on the endothelial cell inflammatory response, HDMEC were exposed to $0.1 \mathrm{mg} / \mathrm{mL}$ HYAPHMB-NC and PLLA-OCT-NP. As a positive control, cells were stimulated with $1 \mu \mathrm{g} / \mathrm{mL}$ lipopolysaccharide derived from E. coli. After 4 (E-selectin) and 24 hours (ICAM-1, VCAM-1, IL-6, IL-8, and MCP-1), EIA and ELISA were performed to analyze the presence of both membrane bound and soluble inflammatory markers.
As can be seen in Figure 6, stimulation of HDMEC with lipopolysaccharide resulted in a significant increase in the expression of the CAMs, ICAM-1, VCAM-1, and E-selectin as well as the cytokines IL-6, IL-8, and MCP-1 (Figure 6) in comparison to unstimulated cells. However, the exposure of cells to HYA-PHMB-NC and to PLLAOCT-NP did not lead to any significant changes in the marker expression profile, implying that no inflammatory response was induced.

\section{Effect of HYA-PHMB-NC and PLLA-OCT- NP on angiogenesis}

To investigate the effect of HYA-PHMB-NC and PLLAOCT-NP on the potential of endothelial cells to form capillary-like structures, HDMEC were co-cultured with HDF. The cultivation period was for 7 days, including an initial 48-hour exposure to $0.01 \mathrm{mg} / \mathrm{mL}$ of each of the nanosamples. To visualize the endothelial cells, the co-cultures were stained with endothelial cell-specific CD31.

Figure 7 shows that the microcapillary-like tube formation by endothelial cells in the co-cultures was not inhibited by the presence of HYA-PHMB-NC (Figure 7A) or by PLLA-OCT-NP (Figure 7B) in comparison to the untreated co-culture (Figure 7C). Microcapillary-like structures were distributed over the entire growth area. Magnification of the image centers (Figure 7Aa-Cc) showed dense and highly branched networks. In the PLLA-OCT-NP-treated co-cultures, the CD31 signal was interspersed with the nanoparticle fluorescence, thus hampering any data quantification. Endothelial microcapillary-like structures were, however, easily distinguished.

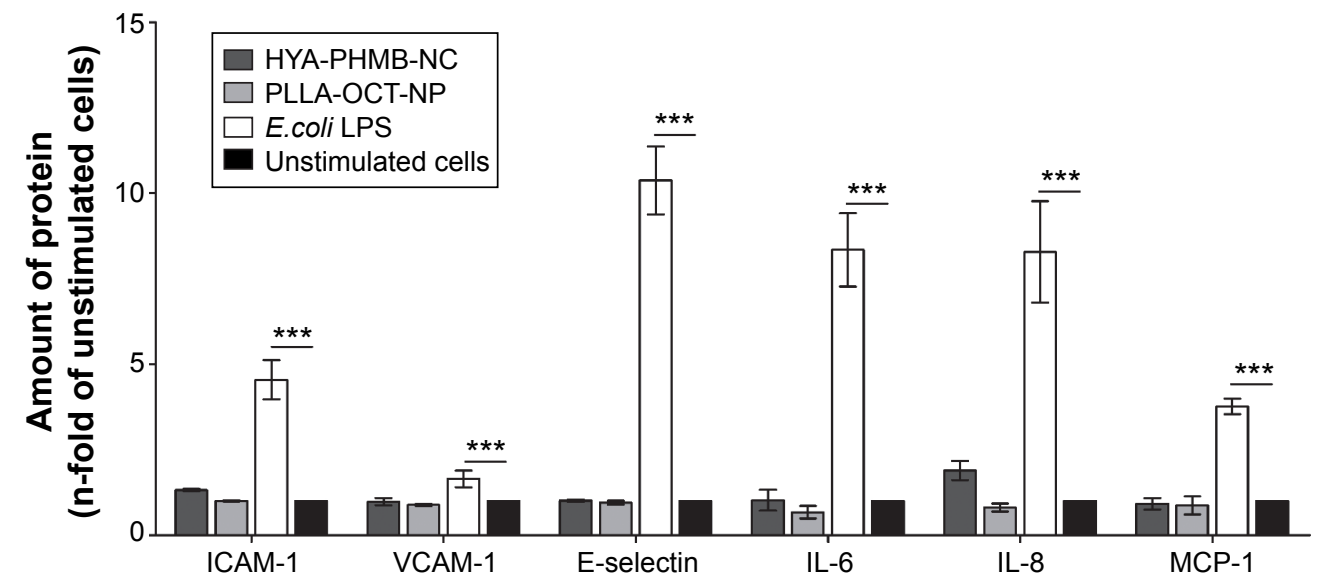

Figure 6 Inflammatory response after stimulation with HYA-PHMB-NC and PLLA-OCT-NP.

Notes: HDMEC were exposed to $0.1 \mathrm{mg} / \mathrm{mL}$ nanosamples for 4 hours (E-selectin) and 24 hours (ICAM-I, VCAM-I, IL-6, IL-8, and MCP-I), before enzyme immunoassay and enzyme-linked immunosorbent assay were performed. One microgram per milliliter Escherichia coli lipopolysaccharide was used as a positive control. $n=3$, $* * * P \leq 0.001$. Abbreviations: LPS, lipopolysaccharide; HYA-PHMB-NC, hyaluronan nanocapsules containing polyhexamethylene biguanide; PLLA-OCT-NP, poly-L-lactic acid nanoparticles loaded with octenidine; HDMEC, human dermal microvascular endothelial cell. 

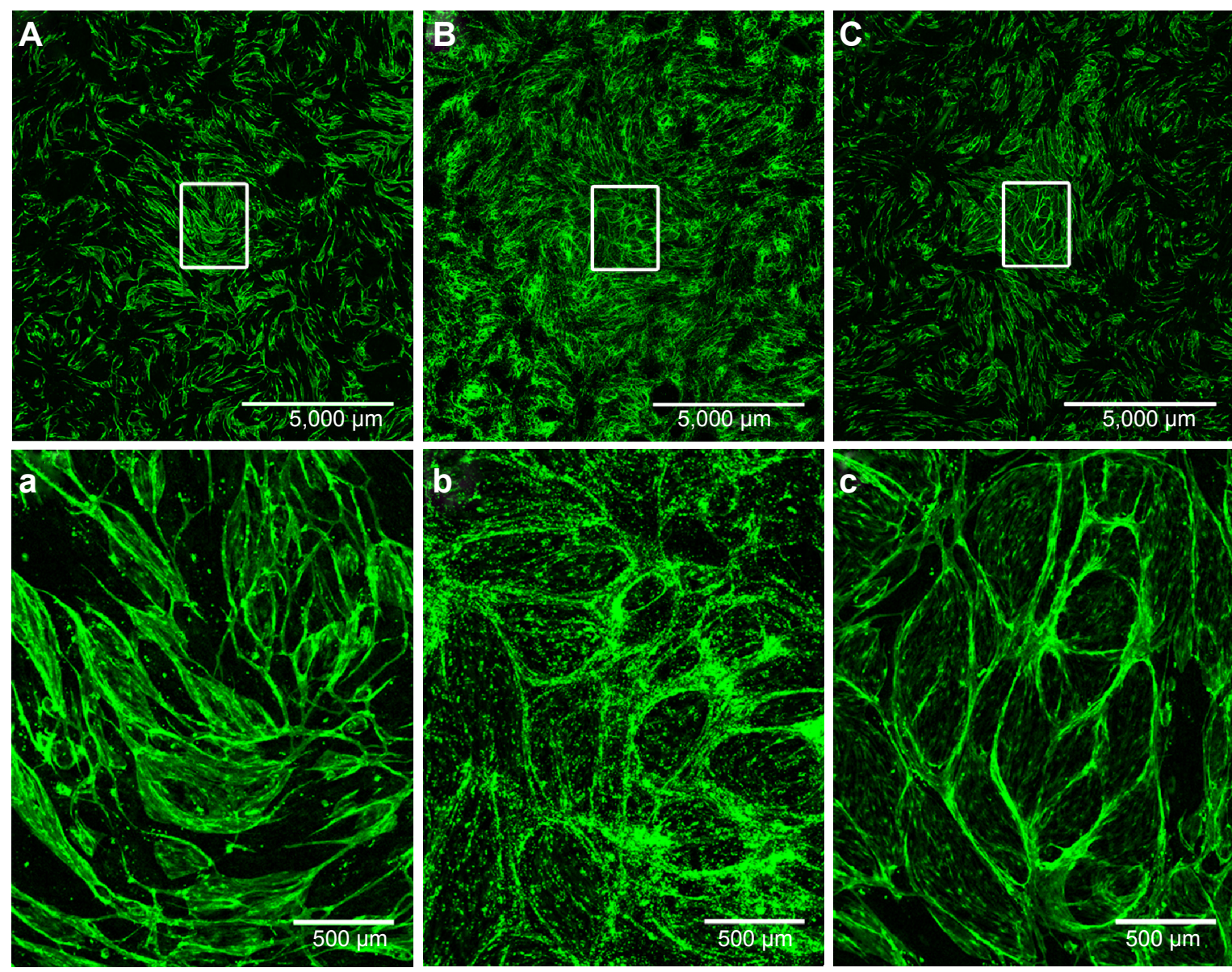

Figure 7 HDMEC/HDF co-cultures after exposure to HYA-PHMB-NC and PLLA-OCT-NP.

Notes: The co-cultures were cultivated for 7 days, including 48 hours exposure to $0.01 \mathrm{mg} / \mathrm{mL}$ HYA-PHMB-NC (A, a) or PLLA-OCT-NP (B, b), before being stained for CD3I (green). Control co-cultures were left untreated (C, c). $n=3$.

Abbreviations: HDMEC, human dermal microvascular endothelial cell; HDF, human dermal fibroblast; HYA-PHMB-NC, hyaluronan nanocapsules containing polyhexamethylene biguanide; PLLA-OCT-NP, poly-L-lactic acid nanoparticles loaded with octenidine.

\section{Discussion}

Bacterial infections represent a serious and frequent problem in wound care. Due to the specific wound characteristics, they often occur in burns, so that prophylactic disinfection measures are generally implemented to lower the risk of infection. However, at the same time these procedures may exhibit substantial negative effects on the tissue and its healing processes. In this study, we investigated two biodegradable, antimicrobial nanosystems and their effects on relevant cells and the process of wound healing. Since they play an important role during various wound healing processes, primary dermal microvascular endothelial cells (HDMECs) were used as an experimental model. The goal of this study was to evaluate the non-degraded nanocarriers with regard to their cytocompatibility and suitability as drug delivery systems in wounds.

By microscopic and flow cytometric analysis after exposure for 2, 8, 24, and 48 hours, we demonstrated that
HYA-PHMB-NC and PLLA-OCT-NP, both labeled and visualized by fluorescent dyes, were internalized by the endothelial cells. For the microscopic investigations, platelet endothelial cell adhesion molecule-1 (CD31), a membrane structural protein, was chosen as an endothelial cell marker to facilitate a precise localization of the internalized materials. The localization of the nanomaterials shown in the microscopic images depended on the exposure time, starting with an unspecific attachment to the cells and ending with a clearly intracellular, perinuclear accumulation after 72 hours of exposure. Image quantification and flow cytometry data also pointed to a time dependency of the rate of uptake for both the nanocarriers. A difference was observed between HYAPHMB-NC and PLLA-OCT-NP in that the internalization process of the latter was already completed after 24 hours, whereas the amount of intracellular HYA-PHMB-NC continuously increased over 72 hours. This might be explained by a different size and/or surface design of the materials, 
which is in accordance with $\mathrm{Nel}$ et al who reviewed the biophysicochemical interactions at the nano-biointerface and the relationship to nanoparticle composition. ${ }^{20}$

Furthermore, we investigated if the nanosystems induced any inflammatory response in endothelial cells. During the inflammatory phase of wound healing, a cascade of events eventually results in the elimination of pathogens and tissue debris and in the proliferation and migration of cells that are involved in the closure of the wound and the regeneration of the healthy tissue. ${ }^{21}$ The expression of three transmembrane CAMs (E-selectin, ICAM-1, and VCAM-1) was examined along with three pro-inflammatory cytokines (IL-6, IL-8, and MCP-1) that are expressed by endothelial cells upon exposure to inflammatory stimuli. ${ }^{22}$ Several other studies have already demonstrated that certain NP with different physicochemical properties can induce inflammatory processes. For instance, for silica NP an inflammatory reaction of the endothelial line ISO-HAS-1 and epithelial cell line H441 was observed in both mono- and co-cultures. ${ }^{23}$ Furthermore, in primary human umbilical vein endothelial cells (HUVECs) the expression of C-reactive protein and the production of pro-inflammatory cytokines were significantly elevated after exposure to silica NP. ${ }^{24}$ In addition, it has been shown that titanium dioxide NP result in an inflammatory cytokine release in lung epithelial cells. ${ }^{25}$ Unlike these nanosystems, HYA-PHMB-NC and PLLA-OCT-NP do not induce a significant inflammatory response. These findings in the present study indicate that vascular inflammatory processes during wound healing will probably not be enhanced or generally interfered with by the presence of HYA-PHMB-NC and PLLA-OCT-NP. Thus, these highly favorable characteristics make these compounds suitable for application in wound treatment.

In addition, we analyzed the impact of HYA-PHMB-NC and PLLA-OCT-NP on the angiogenic potential of endothelial cells by examining the ability of the latter to form capillary-like structures in vitro in the presence of these compounds. Endothelial cells were co-cultured with dermal fibroblasts to mimic the conditions of dermal neovascularization in vivo. Within this co-culture, a spontaneous self-organization of the endothelial cells into tubular vessellike networks can be observed. ${ }^{19}$ Since wound healing and cellular activity require sufficient concentrations of oxygen and nutrients and therefore a sufficient blood circulation, neovascularization is of major importance and a critical step during wound healing. For various types of NP, it has been shown that they can have an inhibitory effect on angiogenesis. Silica NPs have been shown to impair angiogenesis of HUVECs. ${ }^{26}$ Pan et al demonstrated that gold NP inhibited HUVEC VEGF165-induced migration and tube formation. ${ }^{27}$ On the contrary, neither HYA-PHMB-NC nor PLLA-OCT-NP used in the present studies exhibited any effect on the formation of microcapillary-like structures in the NP-treated co-cultures compared to the untreated co-culture. Thus, angiogenesis, one of the most important factors in the wound healing process, is not influenced by the presence of the nanocompounds, further demonstrating their high tissue compatibility.

The cytotoxicity of the nanosystems was assessed by performing both a cell viability and a DNA-staining assay after exposure. Since for an application as responsive drug delivery systems in wounds the effects of a long-term exposure is of particular interest, an exposure time in vitro of 24 hours was generally used as a model of short-term exposure, whereas 7 days were used to mimic long-term exposure. Although uptake analysis demonstrated that a considerable amount of NC or NP, respectively, is internalized, the dose-response curves obtained showed that both the compounds exhibited only mild toxic effects on the endothelial cells when applied at high concentrations. Remarkably, HYA-PHMB-NC and PLLA-OCT-NP affected the cells less than solutions of the antiseptic agents alone when added to the cells at the comparable doses present in the respective NC or NP. These experiments were performed under non-infectious conditions. However, exposure to enzymes that can be produced by common wound germs, eg, S. aureus will lead to the degradation and release of the antiseptic drugs. ${ }^{13,14}$ In a comparative study of antiseptic efficacy, Koburger et al showed that for polyhexanide $0.5-2 \mathrm{mg} / \mathrm{L}$ and for octenidine $2 \mathrm{mg} / \mathrm{L}$ are required for a sufficient inhibition of $S$. aureus. ${ }^{28}$ It should be highlighted that particularly at these concentrations the nanosystems have practically no negative influence on the cells even after 7-day exposure, in contrary to the agents alone. This indicates that unlike a prophylactic antisepsis, wound exposure to the nanosystems will result in negligible effects on cell and tissue viability.

Every injurious stimulus leads to inflammation. Wischke et al observed a significant discrepancy between endothelial cell response upon exposure to different NP under normal and under inflammatory conditions and therefore suggested that inflammatory test conditions should generally be included in in vitro cell studies of nanotoxicity. ${ }^{29}$ However, it is useful to test NP initially under standard conditions in 
order to identify safe and promising candidates. Indeed, all of our experiments were performed using healthy cells under conventional culture conditions. It will be interesting to assess these nanosystems under inflammatory conditions in further studies. In addition, it will also be useful to examine the effects on additional cell types involved in cutaneous wound healing such as fibroblasts and keratinocytes as protagonists of granulation and epithelialization, respectively. Finally, the pathophysiology of wounds remains highly complex, so that further in vivo studies are needed to determine the consequences of an exposure to the nanosystems. However, our data indicate that the HYA-PHMB-NC and PLLA-OCT-NP are highly biocompatible with primary human endothelial cells and important healing processes in vitro and have therapeutic potential for the management of burn wounds.

\section{Conclusion}

The present studies demonstrate that although microscopic and flow cytometric analysis showed time-dependent cellular uptake of HYA-PHMB-NC and PLLA-OCT-NP, none of the nanosystems induced inflammatory response or interfered with endothelial cell angiogenic potential in relevant human cell-based assays in vitro. Furthermore, toxicity assays after short- and long-term exposure demonstrated that both nanocarriers affected the cells less than solutions of the antiseptic agents alone in comparable concentrations. This is encouraging for their application as an innovative drug delivery system with few side effects and a promising alternative to common infection prophylaxis in burn wound treatment.

\section{Acknowledgments}

The authors gratefully acknowledge Anne Sartoris for technical help. This study was supported by the EU FP7 Project Bacteriosafe \#245500.

\section{Disclosure}

The authors report no conflicts of interest in this work.

\section{References}

1. Tompkins RG, Burke JF. Infections of burn wounds. In: Bennet JV, Brachman PS, editors. Hospital Infections. Boston: Little, Brown; 1992:711-730.

2. Kramer A, Daeschlein G, Kammerlander G, et al. Konsensusempfehlung zur Auswahl von Wirkstoffen für die Wundantiseptik [Consensus recommendation for the selection of active substances for wound antisepsis]. Hyg Med. 2004;5:147-157.

3. Hermans MH. A general overview of burn care. Int Wound. 2005;2(3): 206-220.

4. Modak SM, Fox CL Jr. Sulfadiazine silver-resistant Pseudomonas in burns. New topical agents. Arch Surg. 1981;116(7):854-857.
5. Hendry AT, Stewart IO. Silver-resistant Enterobacteriaceae from hospital patients. Can J Microbiol. 1979;25(8):915-921.

6. Dave RN, Joshi HM, Venugopalan VP. Biomedical evaluation of a novel nitrogen oxides releasing wound dressing. JMater Sci Mater Med. 2012;23(12):3097-3106.

7. Klasen HJ. Historical review of the use of silver in the treatment of burns. I. Early uses. Burns. 2000;26(2):117-130.

8. Klasen HJ. Historical review of the use of silver in the treatment of burns. II. Renewed interest for silver. Burns. 2000;26(2):131-138.

9. Rigo C, Ferroni L, Tocco I, et al. Active silver nanoparticles for wound healing. Int J Mol Sci. 2013;14(3):4817-4840.

10. Asha Rani PV, Low Kah Mun G, Hande MP, Valiyaveettil S. Cytotoxicity and genotoxicity of silver nanoparticles in human cells. ACS Nano. 2009;3(2):279-290.

11. Bidgoli SA, Mahdavi M, Rezayat SM, Korani M, Amani A, Ziarati P. Toxicity assessment of nanosilver wound dressing in Wistar rat. Acta Med Iran. 2013;51(4):203-208.

12. Shalumon KT, Anulekha KH, Nair SV, Nair SV, Chennazhi KP, Jayakumar R. Sodium alginate/poly(vinyl alcohol)/nano ZnO composite nanofibers for antibacterial wound dressings. Int J Biol Macromol. 2011; 49(3):247-254.

13. Baier G, Cavallaro A, Vasilev K, Mailänder V, Musyanovych A, Landfester K. Enzyme responsive hyaluronic acid nanocapsules containing polyhexanide and their exposure to bacteria to prevent infection. Biomacromolecules. 2013;14(4):1103-1112.

14. Baier G, Cavallaro A, Friedemann K, et al. Enzymatic degradation of poly(L-lactide) nanoparticles followed by the release of octenidine and their bactericidal effects. Nanomedicine. 2014;10(1):131-139.

15. Agnihotri N, Gupta V, Joshi RM. Aerobic bacterial isolates from burn wound infections and their antibiograms - A five-year study. Burns. 2004;30(3):241-243.

16. Unger RE, Krump-Konvalinkova V, Peters K, Kirkpatrick CJ. In vitro expression of the endothelial phenotype: comparative study of primary isolated cells and cell lines, including the novel cell line HPMECST1.6R. Microvasc Res. 2002;64(3):384-397.

17. van Kooten TG, Klein CL, Wagner M, Kirkpatrick CJ. Focal adhesions and assessment of cytotoxicity. J Biomed Mater Res. 1999;46(1): 33-43.

18. Unger RE, Peters K, Sartoris A, Freese C, Kirkpatrick CJ. Human endothelial cell-based assay for endotoxin as sensitive as the conventional limulus amebocyte lysate assay. Biomaterials. 2014;35(10):3180-3187.

19. Kirkpatrick CJ, Fuchs S, Unger RE. Co-culture systems for vascularization - learning from nature. Adv Drug Deliv Rev. 2011; 63(4-5):291-299.

20. Nel AE, Mädler L, Velegol D, et al. Understanding biophysicochemical interactions at the nano-bio interface. Nat Mater. 2009;8(7):543-557.

21. Eming SA, Martin P, Tomic-Canic M. Wound repair and regeneration: mechanisms, signaling, and translation. Sci Transl Med. 2014; 6(265):265sr6.

22. Bevilacqua MP, Gimbrone MA Jr. Inducible endothelial functions in inflammation and coagulation. Semin Thromb Hemost. 1987;13(4): 425-433.

23. Kasper J, Hermanns MI, Bantz C, et al. Inflammatory and cytotoxic responses of an alveolar-capillary coculture model to silica nanoparticles: comparison with conventional monocultures. Part Fibre Toxicol. 2011;8(1):6.

24. Duan J, Yu Y, Yu Y, et al. Silica nanoparticles induce autophagy and endothelial dysfunction via the PI3K/Akt/mTOR signaling pathway. Int J Nanomed. 2014;9(11):5131-5141.

25. Medina-Reyes EI, Déciga-Alcaraz A, Freyre-Fonseca V, et al. Titanium dioxide nanoparticles induce an adaptive inflammatory response and invasion and proliferation of lung epithelial cells in chorioallantoic membrane. Environ Res. 2015;136(1):424-434.

26. Duan J, Yu Y, Yu Y, et al. Silica nanoparticles enhance autophagic activity, disturb endothelial cell homeostasis and impair angiogenesis. Part Fibre Toxicol. 2014;11:50. 
27. Pan Y, Wu Q, Qin L, Cai J, Du B. Gold nanoparticles inhibit VEGF165induced migration and tube formation of endothelial cells via the Akt pathway. Biomed Res Int. 2014;2014:418624.

28. Koburger T, Hübner NO, Braun M, Siebert J, Kramer A. Standardized comparison of antiseptic efficacy of triclosan, PVP-iodine, octenidine dihydrochloride, polyhexanide and chlorhexidine digluconate. J Antimicrob Chemother. 2010;65(8):1712-1719.
29. Wischke C, Krüger A, Roch T, et al. Endothelial cell response to (co) polymer nanoparticles depending on the inflammatory environment and comonomer ratio. Eur J Pharm Biopharm. 2013;84(2):288-296.

\section{Publish your work in this journal}

The International Journal of Nanomedicine is an international, peerreviewed journal focusing on the application of nanotechnology in diagnostics, therapeutics, and drug delivery systems throughout the biomedical field. This journal is indexed on PubMed Central, MedLine, CAS, SciSearch ${ }^{\circledR}$, Current Contents ${ }^{\circledR} /$ Clinical Medicine,
Journal Citation Reports/Science Edition, EMBase, Scopus and the Elsevier Bibliographic databases. The manuscript management system is completely online and includes a very quick and fair peer-review system, which is all easy to use. Visit http://www.dovepress.com/ testimonials.php to read real quotes from published authors. 